\title{
Territorial aggression can be sensitive to the status of heterospecific intruders
}

\author{
Topi K. Lehtonen ${ }^{\mathrm{a}, \mathrm{b}}$, Jeffrey K. McCrary ${ }^{\mathrm{c}}$, Axel Meyer ${ }^{\mathrm{a}, *}$ \\ a Lehrstuhl für Zoologie und Evolutionsbiologie, Department of Biology, University of Konstanz, Germany \\ ' School of Biological Sciences, Monash University, Australia \\ ' FUNDECI/GAIA, Esración Biológica, Laguna de Apoyo Nature Reserve, Nicaragua
}

\begin{abstract}
A B S T R A C T
Territorial animals are known to be able to differentiate between intruding individuals posing a low or high threat and adjust their aggressive response accordingly. However, plastic territorial aggression based on recognising individuals with different attributes is typically assumed to be relevant only in the context of conspecific interactions. In this study, we investigated territorial aggression of neotropical cichlid fish in their natural habitat to assess whether responses to different types of individuals of another species can also be plastic. We show that arrow cichlids (Amphilophus zaliosus) adjusted their territorial aggression regarding the status of heterospecific intruders: breeding individuals of Amphilophus astorquii received a lower level of aggression than non-breeders. The same pattern was also found for the two different types of $A$. astorquii individuals intruding into conspecific territories. These results suggest that heterospecific individuals should not be ignored when considering selection pressures shaping plasticity of aggressive behaviour in territorial animals.
\end{abstract}

\section{Introduction}

Agonistic behaviour related to territory defence is likely to be costly in terms of energy loss and risk of injury. Therefore, a territory holder may reduce these costs by adjusting its aggression to the level of threat posed by different territory intruders. For example, established neighbours may be treated benignly as compared with strangers (e.g. Backwell and Jennions, 2004). The reduced territorial aggression towards familiar individuals, posing the lowest threat, is often called the 'dear enemy' effect. However, neighbouring territory owners may also represent a higher threat than strangers, depending on the specific environmental or social setting and the type of territory in question (Temeles, 1994; Müller and Manser, 2007). Typically, such patterns of flexible aggression have been considered only in the context of conspecific intruders and territory holders. Hence, despite a relatively large body of literature showing that territorial aggression can be displayed towards heterospecific individuals (e.g. Itzkowitz, 1990; Robinson and Terborgh, 1995; Sedláček et al., 2006; Sturmbauer et al., 2008), these studies of heterospecific interactions have not considered whether territory holders are capable of recognising different types

\footnotetext{
* Corresponding author at: Lehrstuhl für Zoologie und Evolutionsbiologie, Department of Biology, University of Konstanz, 78457 Konstanz, Germany. Tel.: +497531884163 ; fax: +497531883018 .

E-mail address: axel.meyer@uni-konstanz.de (A. Meyer).
}

of individuals belonging to a species other than their own. A question that has therefore remained neglected is whether territory holders should adjust their level of aggression towards different types of heterospecific intruders.

Territorial incursions by heterospecifics, creating a potential for a need to discriminate between different individuals that are not conspecific, are especially likely in communities where many species share the same breeding habitat, as is the case in many aquatic systems with high levels of biodiversity. Accordingly, we examined the occurrence of plastic territorial aggression in Nicaraguan crater lake cichlids of the genus Amphilophus in their natural environment. These fish typically provide biparental care, and during the breeding season, each pair aggressively defends a stationary territory (Barlow, 2000). Several species breed at the same time using partly overlapping habitats, and opportunistically predate on each others offspring, especially when not in a breeding mode themselves (McKaye, 1977; personal observations). We hypothesised that in this type of breeding aggregations, territory owners should benefit by adjusting their territorial aggression not only towards conspecifics but also different individuals of other species, depending on their perceived level of threat. Specifically, we expected that individuals should pose a lesser threat to both conspecific and heterospecific territory holders when they are engaged in breeding (see Barlow, 2000) or are otherwise familiar (Dear enemy: Temeles, 1994) to the territory holders compared to those that are not. We therefore would predict territory holders to respond more aggressively to the latter. 


\section{Materials and methods}

This study was conducted between December 2007 and January 2008 in Lake Apoyo, Nicaragua. This deep, volcanic caldera is filled with water clear enough to allow direct observations of fish behaviour using SCUBA. The study involved two members of the Midas cichlid species complex (see Elmer et al, in press), the recently described Amphilophus astorquii (Stauffer et al., 2008) and a larger species with more pelagic orientation, the arrow cichlid, Amphilophus zaliosus (see Barluenga et al., 2006). We took advantage of a 'natural experiment' created by arrow cichlid territories being intruded by both breeding and non-breeding individuals of $A$. astorquii: we investigated whether arrow cichlids react differently to the two types of heterospecifics. To distinguish between individuals differing in their status, we observed colouration: both sexes of breeding $A$. astorquii are black, sometimes with few yellowish-grey markings with high colour contrast, whereas non-breeding individuals have grey base colouration with dark vertical bars (Fig. 1). Breeders are mainly motivated to take care of their own offspring using previously accumu-

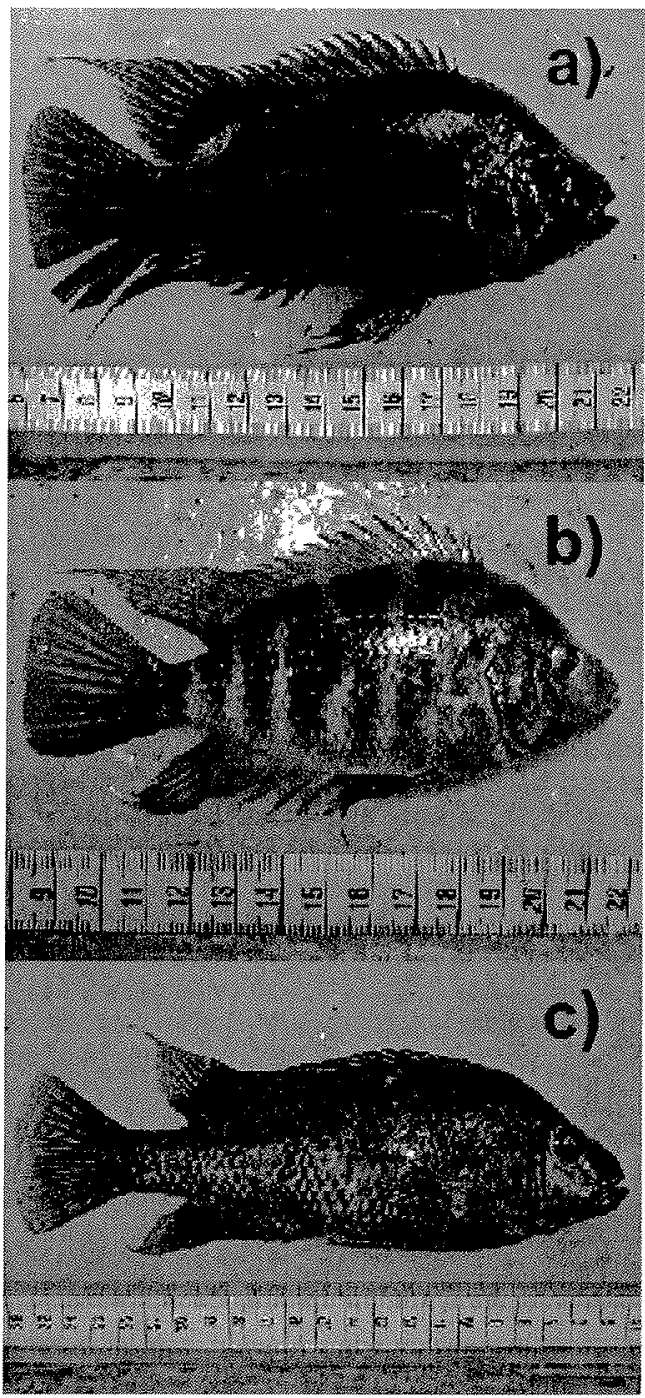

Fig. 1. (a) A. astorquit in breeding colouration, (b) non-breeding $A$ astorquii, and (c) breeding-coloured arrow cichlid (A. zaliosus). lated energy reserves, whereas non-breeding individuals should more likely be looking for food, such as nutritious eggs and juveniles of other cichlids (Barlow, 2000; personal observations). Note, however, that it is likely that the two breeding phase categories were, to some extent, overlapping with familiarity of the individuals ('neighbours' tended to be breeders, whereas 'nonneighbour' intruders tended to be non-breeders). Hence, we cannot currently exclude familiarity as the primary cue for intruder assessment (see Section 4). However, because breeding status is a categorical variable that should be straightforward to assess by both territory holders and human observers, and the way territory holders perceive varying degrees of intruder familiarity is not known, we are using the former as the categorising criterion both in the text and analyses. In order to give a reference point to territorial behaviour of arrow cichlids, we also examined reactions of $A$. astorquii territory holders towards conspecifics belonging to the two breeding phase categories. All non-breeding A. astorquii included in the study were adults, and we did not perceive any size difference between these and breeding individuals.

When a breeding territory was located, the observer approached it up to a distance of approximately $2 \mathrm{~m}$, trying to minimise disturbance. After $3 \mathrm{~min}$, the activities of the territory holders were recorded for $15 \mathrm{~min}$. Each act of aggressive behaviour by the parent fish was classified as being a display, attack or chase. We consider very likely that displays entail lower costs (e.g. energy expenditure) as well as effectiveness (no immediate threat of physical injury to the intruder) than either attacks or chases. Displays, such as flared fins and gills, included only gradual or no movement towards the fish invading the territory and probably only signal awareness of the presence of a potential predator on fry. Attacks were rapid movements towards the intruder until it fled. Some attacks were continued after the intruder had started to flee (a 'chase'). Because of a low prevalence of chases, these were grouped together with attacks for statistical analyses. For each of the aggressive acts, the observer also estimated the distance between the centre of the brood mass and the invading fish. These estimations were calibrated by hav ing a scale on the bottom for the first few replicates for both species.

We observed 10 territories of each of the two species that were subject to incursions by both breeders and non-breeders of $A$ astorquii, allowing paired comparisons between the two groups. Incursions resulting in aggression by territory holders were approximately as common for breeders as non-breeders. It is possible that sometimes territory holders reacted to the same intruding individual more than once. However, as each breeding territory contributed one data point for the paired comparisons between intruding $A$. astorquii breeders and non-breeders, the multiple intrusions did not compromise independence of the data points (behavioural modes: proportion of aggression events that were displays vs. physical aggression; reaction distances: averaged reaction distance over aggression events that occurred during the observation period). We also estimated total lengths of territory holders after having calibrated our approximations by catching a few individuals.

Parametric statistical tests were only applied when their criteria were met. We used repeated-measures ANOVAs to compare the distances of intruders from the centre of the territory at the time territory holders reacted aggressively to them (i.e. reaction distances). The average values for breeders vs. non-breeders for each territory comprised the paired ('repeated') dependent variable and the species of the territory holders was used as a factor. The comparison of the proportions of different behavioural modes was conducted as a non-parametric paired analysis (Wilcoxon signed ranks test). 


\section{Results}

Arrow cichlids exhibited higher average reaction distances towards $A$. astorquii intruders than $\operatorname{did} A$. astorquii territory holders towards intruding conspecifics (repeated-measures ANOVA, species effect, $F_{1,18}=4.49, P=0.048$ ). This difference may have arisen because of the larger size of arrow cichlids (A. astorquii males were estimated as: $17 \pm 0.6 \mathrm{~cm}$ [mean \pm SD], females: $14 \pm 0.8 \mathrm{~cm}$; arrow cichlid males: $26 \pm 1.5 \mathrm{~cm}$, females: $21 \pm 1.9 \mathrm{~cm}$ ), as bigger fish could be expected to defend larger territories. In general, territory holders allowed breeding-coloured $A$. astorquii to approach somewhat closer than non-breeders (Fig. 2), albeit this difference was non-significant (RM-ANOVA, effect of intruder status, $\left.F_{1,18}=3.56, P=0.076\right)$ and independent of the species of the territory holders (RM-ANOVA, intruder status $\times$ species interaction, $F_{1,18}=0.443, P=0.51$; Fig. 2 ).

Territorial behaviours directed towards breeding $A$. astorquii were mostly 'displays', while non-breeding individuals were most of the time immediately attacked or chased away (Fig. 3). This difference in the aggressiveness of territorial behaviour (in terms of proportion of highly aggressive responses) towards the different types of $A$. astorquii individuals was significant both in the case of the arrow cichlid (Wilcoxon signed ranks test, $Z=2.31, N_{1}=N_{2}=10$, $P=0.021$ ) and conspecific territory holders (Wilcoxon signed ranks test, $Z=2.35, N_{1}=N_{2}=10, P=0.019$ ). If only territory holding female arrow cichlids are considered (to rule out any sexual biases in territory defence as the cause of the observed pattern), the conclusion remains the same (Wilcoxon signed ranks test, $Z=2.00, N_{1}=N_{2}=8$, $P=0.046)$.

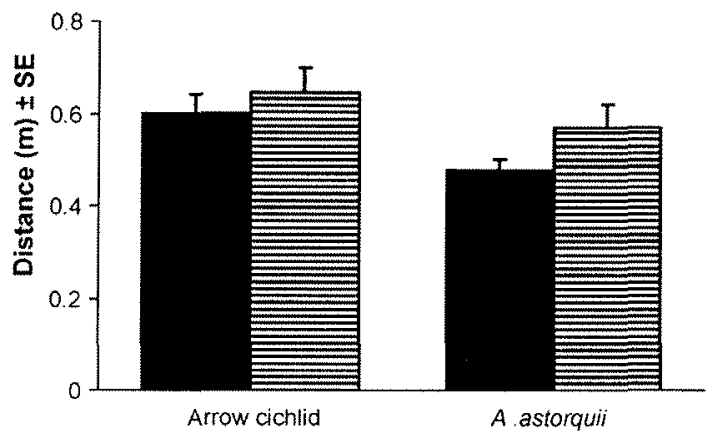

Fig. 2. The distance from the centre of territory at the time of aggressive behaviour Black columns indicate responses towards breeders and columns with horizontal stripes are for non-breeders of Amphilohus astorquii. Sample size for each of the four bars: $N=10$.

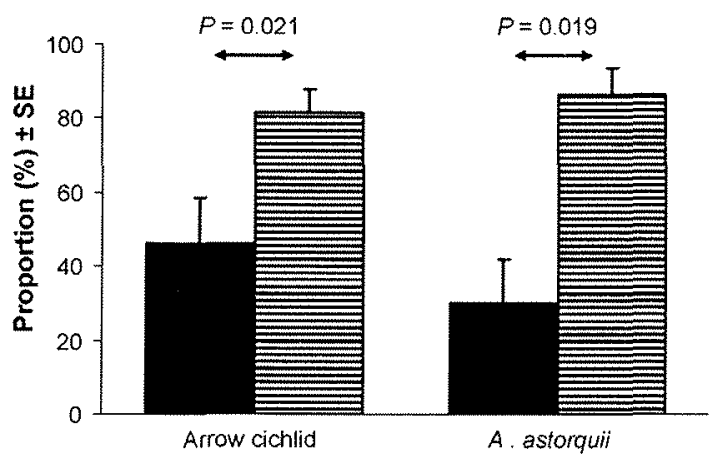

Fig. 3. The proportion of responses with high levels of aggression (attacks and chases) territory holders exhibited towards breeding coloured (black columns) and non-breeding (striped columns) individuals of Amphilohus astorquii. The rest of the responses (100\% minus the proportion shown) were display behaviours. For each of the four columns, $N=10$.

\section{Discussion}

When exhibiting aggressive behaviour towards breeding individuals of $A$. astorquii, territory holding arrow cichlids (A. zaliosus) used threat displays, whereas non-breeding individuals were usually attacked on first sight in the vicinity of the territory. Most of the time territory holders simply ignored $A$. astorquii neighbours that were in breeding colouration, making the difference in response towards the two categories of individuals even larger than our response data suggest. A similar pattern was also found for $A$. astorquii pairs defending their territories towards conspecific intruders. To our knowledge, this is the first study to report plasticity in the level of territorial aggression directed towards different categories of individuals of a species other than their own in any aquatic organism (for a negative result, see Leiser, 2003). However, ants have recently been shown to have the capacity to react differently to heterospecifics from nearby and far away colonies (Tanner and Adler, 2009), which together with our results suggests that the phenomenon could have importance for a wide range of taxa, from insects to vertebrates.

Because $A$. astorquii breeders are preoccupied with defending their own offspring (Barlow, 2000; personal observations), they can be expected to pose a lower threat to territorial arrow cichlids than non-breeding individuals. Hence, arrow cichlids could be using breeding colour or some other cue of reproductive status for distinguishing heterospecific intruders posing different levels of threat. However, it is also conceivable that arrow cichlids are exhibiting a classical form of the 'dear enemy' phenomenon, in which territory residents display lower levels of aggression towards familiar neighbours compared to unfamiliar individuals. In this case, the level of aggression towards established territorial neighbours could be lowered to avoid the costs associated with repeated agonistic encounters, or because these individuals pose a lower risk of territory takeovers than unfamiliar individuals (Temeles, 1994). However, when the territory holder is defending eggs or juveniles, as is the case here, neighbours should pose at least as high a potential for losses as do strangers (Temeles, 1994). Further research is nevertheless needed to differentiate between these two hypotheses.

Even though both conspecific and heterospecific (arrow cichlid) territory holders treated breeding $A$. astorquii individuals with a less aggressive mode than non-breeding individuals, the corresponding difference in reaction distances was not significant. It is possible that predators of offspring (especially Gobiomorus dormitor and juveniles of Parachromis managuensis) are so abundant that parents are forced to hold smaller territories than would be optimal for survival of their current brood. Under this scenario, adjustments to the severity of threat would be achieved more easily with different modes of territorial aggression than by large adjustments to reaction distance.

Arrow cichlids and $A$. astorquii have diverged within Lake Apoyo relatively recently in the evolutionary past (Barluenga et al., 2006). Hence, our results could also be explained by arrow cichlids failing to recognise intruding $A$. astorquii as being heterospecifics. We find this possibility unlikely, because (i) adult arrow cichlids are considerably larger than $A$. astorquii (we found no overlap in within-sex size distributions between the two species), (ii) shape and colouration of the two species are not identical (Stauffer et al., 2008; Fig. 1), and (iii) the two species mate assortatively, suggesting that they do not make mistakes in species recognition. During this study, we did not encounter any heterospecific pairings, or see signs of courtship across the two species.

Here we have shown that arrow cichlids are able to differentiate between individuals of different status even when these belong to another species. We therefore suggest that future studies on individual recognition in the context of territoriality (e.g. dear enemy phenomenon) might like to take into account both conspecific and 
heterospecific individuals, to gain a wider understanding of the costs and benefits that have led to plasticity of aggressive behaviour in territorial animals.

\section{Acknowledgements}

We thank L. López and M. Geiger for the assistance in the field and $\mathrm{B}$. Wong and anonymous reviewers for the helpful comments on earlier versions of this paper. MARENA in Nicaragua issued the necessary permits for the study. Funding was provided by the Alexander von Humboldt foundation (TKL), the Academy of Finland (TKL), the Finnish Cultural Foundation (TKL), the Deutsche Forschungsgemeinschaft (AM) and FUNDECI/GAIA (JKM).

\section{References}

Backwell, P.R.Y., Jennions, M.D., 2004. Coalition among male fiddler crabs. Nature $430,417$.

Barlow, G.W., 2000. Cichlid Fishes: Nature's Grand Experiment in Evolution. Perseus Publishing, Cambridge.

Barluenga, M., Stölting, K.N., Salzburger, W., Muschick, M., Meyer, A., 2006. Sympatric speciation in Nicaraguan crater lake cichlid fish. Nature $439,719-723$.
Elmer, K.R., Kusche, H., Lehtonen, T.K., Meyer, A., in press. Local variation and parallel evolution: morphological and genetic diversity across a species complex of Neotropical crater lake cichlid fishes. Philos. Trans. R. Soc. B. doi: $10.1098 / \mathrm{rstb} .2009 .0271$

Itzkowitz, M., 1990. Heterospecific intruders, territorial defense and reproductive success in the beaugregory damselfish. J. Exp. Mar. Biol. Ecol. 140, 49-59.

Leiser, J.K., 2003. When are neighbours 'dear enemies' and when are they not? The responses of territorial male variegated pupfish, Cyprinodon variegatus, to neighbours, strangers and heterospecifics. Anim. Behav. 65, 453-462.

McKaye, K.R., 1977. Competition for breeding sites between the cichlid fishes of Lake Jiloa, Nicaragua. Ecology $58,291-302$.

Müller, C.A., Manser, M.B., 2007, 'Nasty neighbours' rather than 'dear enemies' in a social carnivore. Proc, R. Soc. B 274, 959-965.

Robinson, S.K., Terborgh, J., 1995. Interspecific aggression and habitat selection by Amazonian birds. J. Anim. Ecol. 64, 1-11.

Sedláček, O., Cikánová, B., Fuchs, R., 2006. Heterospecific rival recognition in the black redstart (Phoenicurus ochruros). Ornis Fennica 83, 153-161.

Stauffer Jr. J.R., McCrary, J.K., Black, K.E., 2008. Three new species of cichlid fishes (Teleostei: Cichlidae) from Lake Apoyo, Nicaragua. Proc. Biol. Soc, Wash. 121, $117-129$.

Sturmbauer, C., Hahn, C., Koblmüller, S., Postl, L., Sinyinza, D., Sefc, K.M., 2008. Variation of territory size and defense behavior in breeding pairs of the endemic Lake Tanganyika cichlid fish Variabilichromis moorii. Hydrobiologia $615,49-56$.

Tanner, C.., Adler, F.R., 2009. To fight or not to fight: context-dependent interspecific aggression in competing ants. Anim. Behav. 77, 297-305.

Temeles, E.J., 1994. The role of neighbours in territorial systems: when are they 'dear enemies'? Anim. Behav. 47, 339-350. 